\title{
THE RESPONSIVENESS OF THE EMORY FUNCTIONAL AMBULATION PROFILE IN REHABILITATION OF AMBULANT STROKE SURVIVORS
}

\author{
Journal website at; \\ http://mrtbjournal.org/index.php/njmr/issue/current/showToc \\ ${ }^{1}$ COAKOSILE, ${ }^{2} \mathrm{VC}$ IGBANUGO, ${ }^{3} \mathrm{BOA}$ ADEGOKE \\ ${ }^{1}$ Department of Medical Rehabilitation, College of Health Sciences, \\ Nnamdi Azikiwe University, Nnewi Campus, Anambra State. Nigeria. \\ ${ }^{2}$ Department of Human Kinetics and Health Education,University of Ibadan. Nigeria. \\ ${ }^{3}$ Department of Physiotherapy, College of Medicine,University of Ibadan and \\ University College Hospital, Ibadan. Nigeria. \\ Correspondence to: \\ COAkosile \\ coakosile@yahoo.com, \\ +234-8028991825
}

\section{SUMMARY}

Background: The Emory Functional Ambulation Profile (EFAP) was designed to measure functional ambulation in post-stroke survivors. Its' ability to detect the effectiveness of any physical therapy protocol has been sparsely investigated.

Objectives: This study aimed to determine the ability of the EFAP to detecting patients' response to a physical therapy protocol in stroke rehabilitation.

Methods: The pretest-posttest experimental design was used for this study. Seventeen consecutive stroke survivors who met the inclusion criteria were recruited into the study. Participants received a conservative physical therapy protocol twice weekly for 8 weeks. Performance on individual subtasks of the EFAP were measured and recorded for each participant before and at the end of the study. Data was available at the posttest for only 14 participants (mean age $=57.009 .05$ years; average poststroke period $=19.7126 .56$ months) and this was analyzed using frequency and percentages with inferential statistics of paired t-test at .05 alpha level.

Results: Participants scores for all the EFAP subtasks and overall scores improved (reduced task completion time) at the end of the treatment programme. Responsiveness for the EFAP ranged from $3 \%$ to $21 \%$ for all subtasks. Changes were even significant for three [floor carpet, up and go] out of the 5 subtasks and the total EFAP scores $(\mathrm{p}<.05)$
Conclusion: EFAP was able to detect the response of stroke survivors to the physical therapy protocol used in this study and is therefore recommended for use by clinicians and researchers for measuring treatment outcome.

Keywords: EFAP, Stroke, Physical Therapy, Responsiveness, Functional ambulation.

\section{INTRODUCTION}

Assessments are often undertaken to predict the ability of individuals to use a variety of skills when performing tasks necessary for daily living, leisure, vocational pursuits and other required behaviours (Eisenberg et al, 1995). Different authors have contended that any assessment of ambulation should include environmental variables such as different terrains, obstacles and stairs ((Cohen et al, 1987; Robinnet and Vondran, 1988; Means, 1996). Wolf et al (1999) reported Fox et al (1996) as having highlighted the need to assess successful ambulation which contributes to meaningful activity. They then defined functional ambulation as the ability of a person to walk with maximal independence and in the least time under various environmental circumstances.

Measures of gait have been used in both laboratory and clinical settings (Mathias et al, 1996) Laboratory gait analyses may be useful because quantitative measures included in such analyses, including decreased speed and decreased stride length have been associated with increased risk of falling (Thorban and Newton, 1996). These laboratory tests often require electronic equipment such 
as electrogoniometers, electrodes footswitches, computers and video cameras (Turnbull and Wall, 1985; Wolfson et al, 1990; Shumway-Cook et al, 1997). These technical gait analyses may be costly and time-consuming, requiring extensive training to administer and often do not assess walking across commonly encountered terrains (Wolf et al, 1999).

Wolf et al (1999) opined that some more clinically applicable tests of ambulation are easy to administer and require only a stopwatch but cautioned that both laboratory gait analyses and simple tests of gait speed typically do not test the ability to move around obstacles over different, surfaces. Nelson (1974) first described a functional ambulation profile. He marked the plantar surface of the foot or shoe and recorded foot contact aspects of gait such as stride length, cadence and others. Wolf (1979) designed the ambulation profile now known as the Emory Functional Ambulation Profile (EFAP), which is a timedtest comprising five subtasks-(floor, carpet, up and go, obstacle and stairs). The EFAP's reliability and validity in measuring walking time was only established later by Wolf et al (1999). A yet to be published study had also established the validity and reliability of the instrument in the Nigerian setting. The use of EFAP as an outcome measure demonstrating the effectiveness of any physical therapy protocol has been sparse and probably restricted to the recent study by Embrey at al (2010)]. An establishment of its responsiveness to detecting changes with different therapy protocols may have positive consequences for its use in research and in the clinics. This study aimed to determine the ability of the EFAP to detect response to a physical therapy protocol using a protocol commonly used by clinicians for stroke management in the authors' local environment.

\section{Methodology}

The research design for this study was the pretestposttest experimental design. The poststroke individuals had their pretest EFAP scores taken at the beginning. Posttest measurements were taken after the 16th treatment session (or after 8 weeks). The joint Institutional Review Committee of UI/UCH gave approval for this study.

The population for this study comprised all male and female poststroke individuals referred for physiotherapy at U.C.H., Ibadan and R.R.S.H., Ibadan. Seventeen (17) consecutively referred volunteer poststroke individuals who matched the inclusion criteria (which included signing of a written informed consent) were recruited into the study as they became available. The inclusion criterion for this study was that participants must be able to perform the five subtasks on the Emory Functional Ambulation Profile (EFAP) without any form of human assistance but with the use of assistive device (if required). The presence of musculoskeletal or other cardiovascular problem apart from the known and controlled hypertension excluded an individual from the study.

\section{Research Instrument}

1. Emory Functional Ambulation Profile (EFAP). Five substasks comprised the EFAP:

i. a 5-meter walk on the hard surface floor marked out on the gymnasium floor.

ii. a 5-meter walk on the carpeted floor

iii. performance of 'up and go' task

iv. negotiation of an obstacle course

v. ascent and descent of 4 stairs

Subtasks were completed in the above sequence (a standardized protocol).

Each participant was given a rest period between performances long enough for one of the researchers (ACO) to explain and demonstrate the next component (average of about 3 minutes). Two of the researchers (ACO and $\mathrm{ABOA}$ ) recorded the performance times for all five subtasks and calculated the total EFAP score on completion of the entire data collection session. Baseline and post-test measurements were taken on days preceding the commencement and immediately after the completion of intervention programme respectively. This ensured there was no interaction of fatigue with either the intervention or measurement. There was also no mid-course measurement to avoid as much as possible the occurrence of learning effect. The average score of two measurements taken prior to intervention and also after completion of intervention served as baseline and post-test measurements respectively. Average score recorded for each test performance by the two raters were taken for each participant. The scores were however not often different between raters and when they do, were not more than 0.5 seconds apart.

The EFAP has a high intrarater $(\mathrm{ICC}=.997)$ reliability as reported by Wolf et al (1999). The EFAP also has a concurrent validity of $r=-.708$ and $r=-.783$ (with and without assistance factor respectively) for poststroke individuals when compared with the Timed 10 metre Walk Test and $\mathrm{r}=-602$ and $\mathrm{r}=-.592$ (with and without assistance factor respectively) when compared with the Berg Balance Test (Wolf et al, 2001).

\section{The Conservative Physical Therapy (CPT) Protocol}

The conservative physical therapy (CPT) protocol was given by two experienced physical therapists working in the hospitals that served as research venues.

Cycle Ergometry: Each participant was made to cycle at zero or maximal comfortable load (which is then noted) for as long as possible before fatigue sets in or maximally 20 minutes. Increments were made with $15 \mathrm{w} / \mathrm{min}$ once the participant adjusted adequately to pre-set load. The cycle ergometer programme followed immediately after the manually-resisted active exercises programmes. Some participants initially had the paretic foot bandage to the foot pedal to avoid slipping off.

Gait Training: Participants were made to walk to and fro on a $5 \mathrm{~m}$ long Frenkel's mat to improve their walking coordination. Each participant moved at comfortable walk 
speeds similar to the speed used for EFAP testing. They did this for as long as they can but not exceeding 15 minutes at each treatment session.

For ethical reasons the upper limb rehabilitation needs of the study participants were not neglected and included the following:

Functional Board training: The Functional board used in this study had ports for (i) weaving (ii) tying and untying shoe buckler, (iii) open and closing door with a Union key, (iv) locking and unlocking a padlock. These were included so that the upper limb's functional needs were not neglected and ensure participants complete treatment though functional ambulation was the aim of the study. The time for training was 15 minutes.

Reach Activities for upper arm function in sitting and standing: These were divided into 2 stages:

(1) While sitting on a chair, each participant reached out to pick an object placed on a table some distance away (far enough to allow reach and also shift some weight off the buttocks) and brought it close to the mouth. The position was reset after each trial up to a maximum of 5 minutes.

(2) While standing, participants continually reached up to touch a pre-determined spot above their head but within their expected reach. This was done for 10 minutes.

\section{Manually-resisted Active Exercises}

While lying on a plinth, each participant was asked to lift up his or her affected upper limb from the shoulder towards the head against the researcher's resistance. This was done repeatedly for 10 times. The participant was then asked to lift up his or her affected lower limb from the hip against the researcher's resistance. This was also done for 10 times. Both activities were repeated for 3 times each.

Data Analysis: The Statistical Package for Social Sciences (SPSS) version 11 was used for data analysis in this study. The mean, standard deviation, minimum and maximum values for each test score, age and for time since onset of stroke (poststroke period) was determined. The mean response for each subtask and overall EFAP was calculated as the difference between the mean pretest score and the posttest score. Percentage response for each subtask and overall EFAP was then calculated using the formula: mean response score /mean pretest score $\times 100$. Gender of poststroke individuals and side of lesion was summarized as frequency occurrence. Paired t-test was used to compare differences between pretest and posttest EFAP scores. Alpha level was set at .05 for all tests.

\section{RESULTS}

Fourteen $(82.4 \%)$ of the study's participants completed the study. The participants had a mean age of 579.05 years and an average post-stroke occurrence period of 19.7126 .56 months. After 8 weeks of treatment, the mean response (mean pretest minus posttest scores) in seconds and percentage change to the physical therapy protocol were $3.45(21.20 \%), 3.35(18.39 \%), 6.07(20.31 \%)$, $6.26(13.07 \%)$ and $0.68(3.25 \%)$ for the floor, carpet, up and go, obstacle and stairs subtasks respectively. The overall mean response and percentage change in EFAP scores were 19.82 seconds and $14.88 \%$ respectively. The posttest scores were significantly lower than the mean pretest scores at .05 alpha level for the floor, carpet and up and go subtasks respectively (Table 1). The mean posttest (task completion times) scores for the obstacle and stairs subtasks were lower than the mean pretest scores but not significantly. However, the mean overall or total EFAP score showed significant reduction $(\mathrm{p}<.05)$ after 8 weeks of treatment (Table 1).

Table 1: Paired t-test for Comparison of Participants' Scores Pre- and Post -Test

\begin{tabular}{lllll}
\hline Subtask & $\begin{array}{l}\text { Pretest } \\
\mathbf{x}+\text { S.D } \\
\text { (Seconds) }\end{array}$ & $\begin{array}{l}\text { Posttest } \\
\mathbf{x}+\text { S.D } \\
\text { (Seconds) }\end{array}$ & t & P \\
\hline Floor & 16.27010 .278 & 12.8158 .270 & 4.654 & $0.000^{*}$ \\
Carpet & 18.22312 .947 & 14.8669 .242 & 2.776 & $0.016^{*}$ \\
Up and Go & 29.89016 .400 & 23.82411 .520 & 3.978 & $0.002^{*}$ \\
Obstacle & 47.88828 .574 & 41.62727 .057 & 1.887 & 0.082 \\
Stairs & 20.9328 .857 & 20.2509 .608 & 0.867 & 0.402 \\
Total & 133.20174 .607 & 113.38362 .646 & 3.768 & $0.002^{*}$ \\
\hline df $=12$ & & & & \\
$t($ critical) $=2.160$ & & & \\
$*=$ significant at $\mathrm{p}<0.05$ & & &
\end{tabular}

\section{DISCUSSION}

The percentage response scores for participants ranged between $13 \%$ and $21 \%$ for the overall EFAP except the stairs subtask suggesting that the EFAP was fairly responsive to the 8-week physical therapy protocol used in this study. EFAP responses on its floor, carpet and up and go subtasks were significantly better after 8 weeks for study participants while statistical significance could not be established for its responses on the obstacles and stairs subtasks. The floor, carpet and up and go subtasks are all level walking activities and require lesser degree of dynamic balance than the other two. Those EFAP subtasks with lesser balance requirements seem better able to show overtime changes occurring due to physical therapy intervention. Obstacles and stairs tasks, on the other hand, are activities which are more challenging than level walking. Between the two however, EFAP was far more responsive on the obstacle subtask than it was on the stairs. Brown et al (2005) had shown that greater attentional demand is required pre- and during obstacle crossing and that a greater risk of falling exists with the task especially with older adults. Said et al (1999) had suggested that this threat to gait safety is particularly higher in stroke population as a result of the findings that the ability to negotiate obstacles was compromised and inconsistent in their studied group. Troy and Grabiner (2005) opined that quick restoration of dynamic balance is requisite for 
the obstacle task. Cromwell and Wellmon (2001) and Nadeau et al (2003) had highlighted the increased challenge of stair ascent and descent compared to level walking. The direction of body segment changes to maintain balance in stairs task and kinematics and kinetics are considerably higher for stairs task than for level waking (Cromwell and Wellmon, 2001). Our findings suggest this to be true even in instances where the level walking requires the negotiation of an obstacle course. The non-inclusion of the balance activities for study participants in this study might be responsible for the marginal and non-statistically significant improvement obtained in the stairs subtask, especially when one considers that there was a remarkable reduction $(15 \%)$ in their total EFAP scores, which implies an improved average walking speed across tasks. It seems that for the stairs subtasks of the EFAP to be sensitive to detect clinically reasonable and statistically significant changes with a therapy protocol for stroke, such protocol must include activities targeting balance. Geiger et al (2001) had shown reported improvement in Berg balance and "Timed up and go" scores with a conservative physical therapy protocol which included series of balance activities in addition to the activities in the CPT protocol of this study. The inclusion of such balance activities to the CPT protocol of the study may further aid the ability of the stairs subtask of the EFAP to detect changes in stroke survivors who are receiving such treatment over a particular time period. It might also be that the EFAP stairs subtask might be weak in detecting survivors' responses to a physical therapy protocol considering the fact that the percentage response at 3.25 was far lower than what obtained for the other subtasks. This study did not look at the effect of time on the ability of EFAP to detect patients' response to treatment. It may not be impossible for even the CPT protocol used in the study to give remarkably higher changes even in the survivors' stairs climbing ability with a longer time frame. It is however important to emphasize that the slightly above $3 \%$ change in the stairs subtask might be more important for the stroke survivor than could be imagined. French (1998) had cautioned that there is a need to distinguish between clinical/practical and statistical significance and that a little and statisticallyinsignificant change in outcome measure might have farreaching clinical implications.

The increased walking speed for the study's participants may be presumed to be due to improvements in cadence of gait and stride length. The relationship between these variables had previously been established (Perry et al, 1995; Roth et al, 1997). Changes in gait cadence and stride length as a result of a therapeutic intervention may just also be reflecting changes in the ability to ambulate functionally, particularly at the community level. Improvement in walking has been reported to naturally lead to an improvement in the individual's ability to walk independently confidently (David et al, 2004). There is however a need for the relationship between these variables and the EFAP subtasks to be studied. Guimares and Isaacs (1980) had identified walking speed improvement as an indicator for reduced risk of falls and subsequent injury. The EFAP more or less assesses the speed of walking in various circumstances, thus an individual's EFAP scores might also just indicate his/her likelihood of having a fall or injury. Kwakkel and Wagenaar (2002) had shown that the increased stability and asymmetry of walking resulting from improved walking speed is better observed when individuals move at comfortable walking speed after stroke. All EFAP subtasks involve movement at comfortable walking speeds. David et al (2004) opined that this ability to walk safely at a functional speed would enhance the quality of life of poststroke individuals by promoting increased involvement with family and community. The EFAP as a therapeutic outcome measure may also be an indicator of the stroke survivors' quality of life and level of social participation. We suggest that instruments measuring these two variables should also be correlated with the EFAP.

\section{CONCLUSION}

The study showed EFAP's to be responsive in detecting changes among stroke survivors being managed with a physical therapy protocol especially in those subtasks requiring lesser degree of balance. It is thus recommended for clinicians managing stroke survivors who are interested in having a valid but simple and readilyavailable outcome measuring tool for evaluating the effectiveness of their intervention.

\section{ACKNOWLEDGEMENT}

The authors would like to thank Bukky Magbaralewon for typing the manuscript.

\section{REFERENCES}

- Bohannon RW (1987). Gait performance of hemiparetic stroke patients: selected variables. Archives of Physical Medicine and Rehabilitation. 68:777-781.

- Brown LA, McKenzie NL and Doan JB (2005). Age dependent differences in the attentional demands of obstacle negotiation. Journal of Geriatric Science and Medical Sciences 60(7):924-927.

- Cohen JJ, Sween JD, Walker JM and Smith KB (1987). Establishing criteria for community ambulation. Topics in Geriatric Rehabilitation. 1987. 3:71-77.

- Cromwell R and Wellmon R (2001). Sagital plane head stabilization during level walking and ambulation on stairs. Physio Research International 6 (3):1799-192.

- David DL, Brown DA, Pierson-Carey CD, Buckley EL and Lew HL (2004). Stepping over obstacles to improve walking in individuals with poststroke hemiplegia. Journal of Rehabilitation Research and Development. 41(3A):283-292.

- Eisenberg MG (1995). Dictionary of Rehabilitation. New York. Springer Publishing Company. PP 103 
- Embrey DG, Holtz SL, Alon G, Brandsma BA, McCoy SW (2010) Functional electrical stimulation to dorsiflexors and plantarflexors to improve gait during walking in adults with chronic hemiplegia. Archives of Physical Medicine and Rehabilitation 91(5): 687-696

- French S (1998) How significant is statistical significance? A critique of the use of statistics in research. Physiotherapy. 74: $266-268$

- Geiger RA, Allen JB, O'Keefe J and Hicks RR (2001). Balance and mobility following stroke: effects of physical therapy interventions with and without biofeedback/ forceplate training. Physical Therapy 81:995-1005.

- Guimares RM and Isaacs B (1980). Incidence and consequences of falls due to stroke: a systematic inquiry. International Rehabilitation Medicine. 2: 177-80.

- Kwakkel G and Wagenaar RC (2002). Effect of duration of upper- and lower- extremity rehabilitation on sessions and walking speed on recovery of interlimb coordination in hemiplegic gait. Physical Therapy. 82: 432-448.

- Mathias S, Nayak USL and Isaac B (1996). Balance in elderly patients: the get up and go test. Archives of Physical Medicine and Rehabilitation. 67:387-389.

- Means KM (1996). The obstacle course: a tool for the assessment of functional balance and mobility in the elderly. Journal of Rehabiltation Research. 33:413-429.

- Nadeau S, McFadyen BJ and Malouin F (2003). Frontal and sagital plane analysis of the stairs climbing task in healthy adults aged over 40 years: what are the challenges compared to level walking? Clinical Biomechanics. 18(10):950-959.

- Nelson AJ (1974). Functional ambulation profile. Physical Therapy. 54:1059-1065.

- Perry J, Garret M, Gronely JK and Mulroy SJ (1995). Classification of walking handicap in stroke population. Stroke. 26:982-989.

- Robinett CS and Vondran MA(1988). Functional ambulation velocity and distance requirements in rural and urban communities: a clinical report. Physical Therapy. 61:351353.
- Robinson JL and Smidt GL (1981). Quantitative gait evaluation in the clinic. Physical Therapy. 61: 351-353

- Roth EJ, Merbitz C, Mroczek K, Dugan SA and Suh WW (1997). Hemiplegic gait relationship between walking speed and other temporal parameter. American Journal of Physical Medicine and Rehabilitation. 76(2): 128-133.

- Said CM, Goldie PA, Patla AE, Sparrow WA and Martin KE (1999). Obstacle crossing in subjects with stroke. Archives of Physical Medicine and Rehabilitation. 80(9):1054-1059.

- Shumway-Cook A, Baldwin M, Polissar NL and Gruber W (1997). Predicting the probability of falls in communitydwelling older adults. Physical Therapy. 77:812-819

- Thorbahn LD and Newton RA (1996). Use of the Berg Balance Test to predict falls in elderly persons. Physical Therapy. 76: 576-585.

- Tinetti ME and Ginter SF (1988). Identifying mobility dysfunctions in elderly patients, standard neuromuscular examination or direct assessment? Journal of the American Medical Association. 259:1190-1193.

- Troy KL and Grabiner MO (2005). The presence of an obstacle influences the stepping response during induced trips and surrogate tasks. Experimental Brain Research. 161:343-350.

- Turnbull GI and Wall IC (1985). The development of a system for the clinical assessment of gait following a stroke. Physiotherapy. 71(7): 294-298.

- Wolf SL, Catlin PA, Gage K, Gurucharri, K, Robertson R and Stephen K (1999). Establishing the reliability and validity of measurement of walking time using the Emory functional ambulation profile. Physical Therapy. 79:11221133.

- Wolf SL. (1979) A method for quantifying ambulatory activities. Physical Therapy. 59:767-768.

- Wolfson L, Whipple R, Amerman P and Tobin JN (1990). Gait assessment in the elderly: a gait abnormality rating scale and its relation to falls. Journal of Gerontology. 45:M12-M19. 